\title{
Optimizing Residual Plots for Likert Data
}

\begin{abstract}
Likert-type questions are widely used in survey in social science and produce discrete and repeated data. When plotting residuals from a linear model whose dependent variable is measured by Likert-type question, researchers might have problem observing the plot which is always with parallel lines. Adding some disturbance to the dependent variable before plotting can optimize the plot and solve this problem.
\end{abstract}

It is still debatable whether Likert type data can be applied in parametric statistical process such as Linear Regression and Factor Analysis. A typical Likert scale item has 5 to 11 points that indicate the degree of agreement with a statement, such as 1=Strongly Agree to 5=Strongly Disagree. It can be a 1 to 5 scale, 1 to 7, etc. (Grace-Martin, 2008) One camp maintains that as ordered categories, the intervals between the scale values are not equal. Any mean, correlation, or other numerical operation applied to them is invalid (Jamieson, 2004). The other camp maintains that while technically the Likert scale item is ordered, using it in parametric tests $i s$ valid in some situations. Grace-Martin (2008) points out at the very least, insist that the item have at least 5 points ( 7 is better), that the underlying concept be continuous, and that there be some indication that the intervals between points are approximately equal. Researchers should also make sure the other assumptions (normality \& equal variance of residuals, etc.) be met.

Residual plots are commonly made to check for homoscedasticity of errors or presence of nonlinearity in the systematic part of the model (Nelder, 1990). If the model does NOT meet the linear model assumption, we would see our residuals take on a defined shape or a distinctive pattern. To assess if the homoscedasticity assumption is met, we look to make sure that the residuals are equally spread around the $y=0$ line. Searle (1988) indicated that when observed values of $y$ are repeated in the data, the plot of residuals against fitted values would exhibit a series of parallel lines having slope -1 . The reason is the plot is of $y-\hat{y}$ against $\hat{y}$. Consider for $\mathrm{y}$ of some value $\mathrm{c}$, then it is of $(y-\hat{y}) \mid y=c$ against $(\hat{y}) \mid y=c$, i.e. $(c-(\hat{y}) \mid y=c)$ against $(\hat{y}) \mid y=c$. So, it is a straight line with slope of -1 and intersect of $c$.

Due to this reason, when Likert type question is used for mearing a dependent variable, the residual plot for the linear model would always be parallel lines with slopes of -1 and intersect of values of Likert data. These characteristics make researchers difficult to observe the pattern as what they did in other continues data. So, it might be useful to optimize the residual plot when it involves Likert type data. 
To optimize the residual plots, we add a disturbance term to dependent variable before plotting. And we will show its usefulness in the simulations below. The first simulated data is generated by linear model $1 \mathrm{y}=2+0.4 * \mathrm{X}+\mathrm{e}$. To turn it to Likert data, $\mathrm{y}$ is then rounded. Values of $\mathrm{y}$ smaller 1 are given 1, and values of y larger than 7 are given 7 . So, the new values of $y, y^{\prime}$, range from 1 to 7 discretely and fit a nearly linear model. We then fit the data to a linear model (Table 1) using " $1 \mathrm{~m}$ " function in R and plot the residual plot. This plot is y' - fitted against fitted. The plot has 7 parallel lines as we explained above. If we plot $y$ - fitted against fitted, it will be a plot without pattern and nearly normally distributed in the cross section which corresponds to linearity and homoscedasticity assumption. However, we do not know the true value in real life, so one way to simulate the true value is to add a uniform distributed term to y'. Here, a disturbance term $\mathrm{d} \sim \mathrm{U}(-.5,5)$ is added to $\mathrm{y}^{\prime}$ and we got $\mathrm{y} \_$new as a result. The plot of y_new fitted against fitted is like y - fitted against fitted and help us to check linearity and homoscedasticity assumption. (Figure 1)

The second simulated data is generated by quadratic model $2 \mathrm{y}=5-1 * X+0.1 * X * * 2+\mathrm{e}$. Like the first simulation, data are mapped to 1 to 7 discretely. The " $1 \mathrm{~m}$ " function also indicates its coefficient for linear model is significant (Table 1) which is not true. Its y'-fitted against fitted plot has pattern indicating the unsatisfatory of linearity assumption and y_new-fitted against fitted also shows the similar pattern (Figure 1).

These simulations show that adding disturbance term to the dependent variable measured by Likert type question and re-plotting the residual plots can help researchers to check the assumptions of linear models.

Table 1: Coefficients of model 1 and model 2.

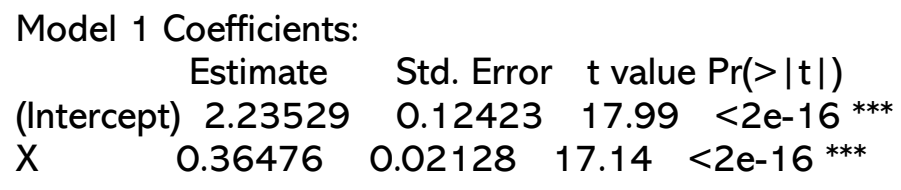



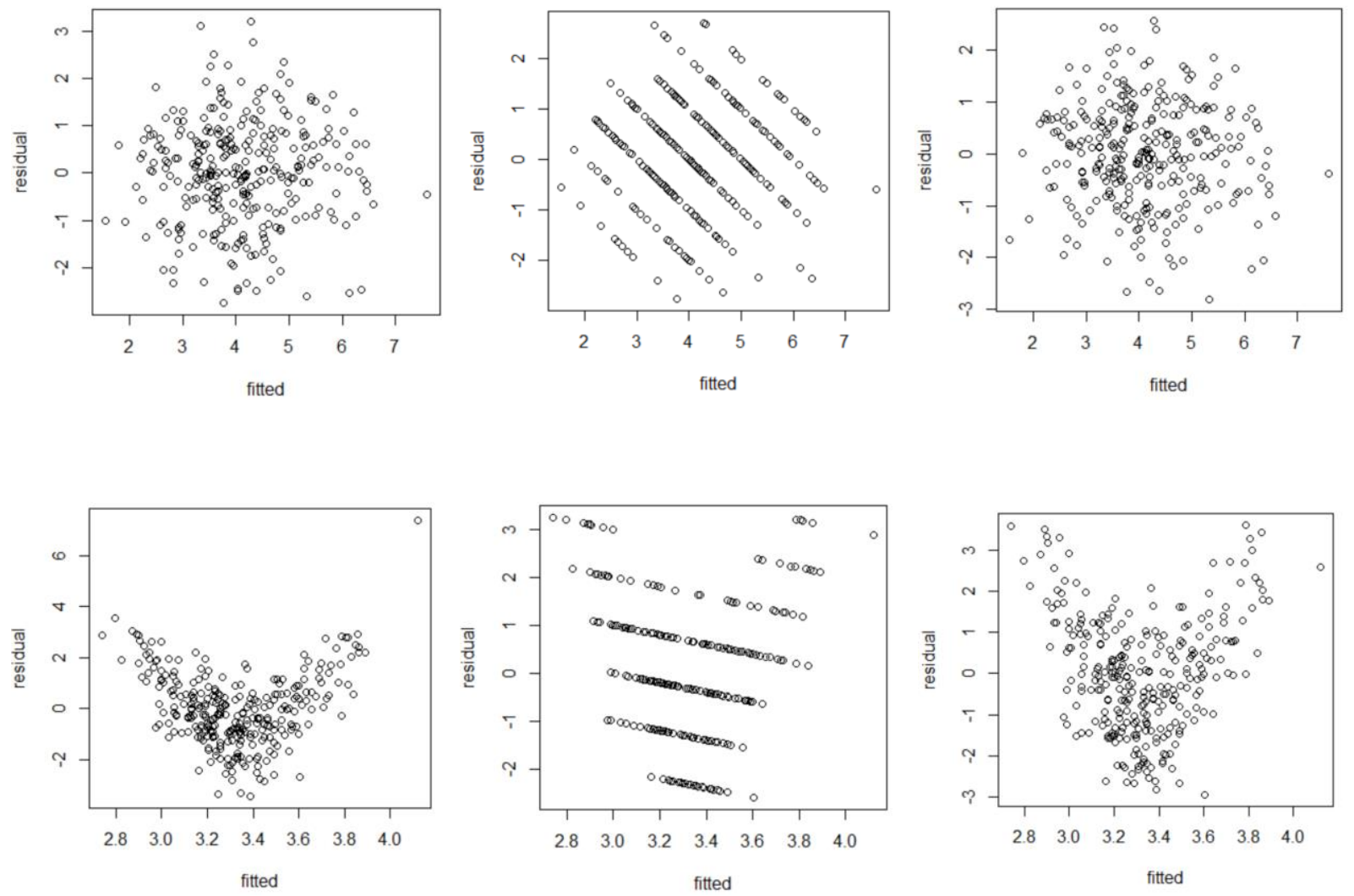

Figure 1: The residual plots of model 1 and model 2.

The first row is for model 1, the second row is for model 2. From left to right is plot for y'-fitted

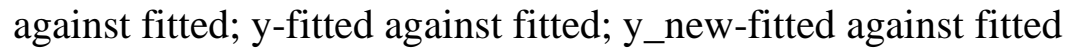

\section{References}

Grace-Martin, K. (2008). Can Likert scale data ever be continuous. Article Alley. Jamieson, S. (2004). Likert scales: How to (ab) use them?. Medical education, 38(12), $1217-$ 1218.

Nelder, J. A. (1990). Nearly parallel lines in residual plots. The American Statistician, 44(3), 221-222.

Searle, S. R. (1988). Parallel lines in residual plots. The American Statistician, 42(3), 211-211. 


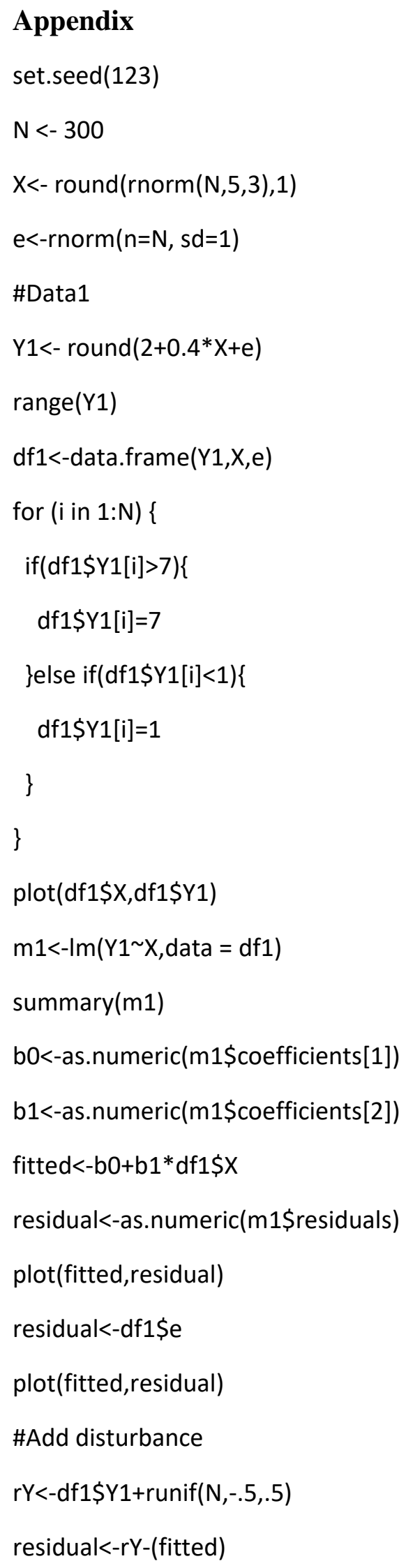




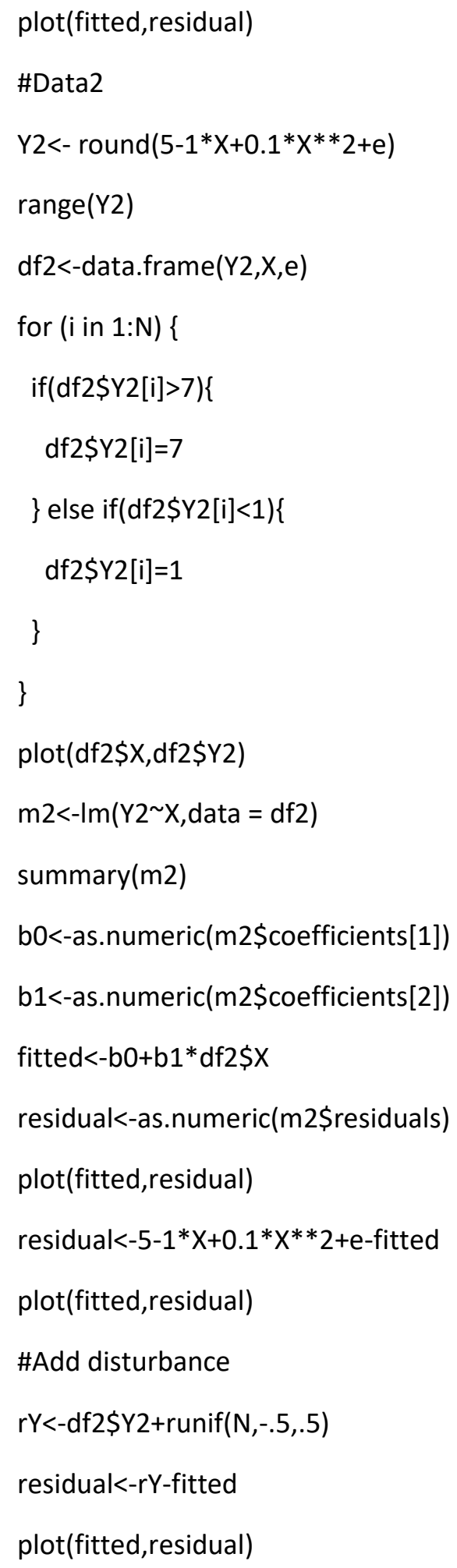

